\title{
BALANCE MOTION PLANNING FOR HUMANOID ROBOT BASED ON 3D INVERTED PENDULUM MODEL
}

\author{
DUONG MIEN KA, TRAN HUU TOAN \\ Journal of Science and Technology, Industrial University of Ho Chi Minh City; \\ duongmienka@gmail.com
}

\begin{abstract}
Researches on humanoid robots are alway attractive to many researchers in robotics field. One of considerable challenges of humanoid robots is to keep balance and stability of their movement. Because a humanoid robot moves by two legs, most of time of the step period of the humanoid robot is be in one leg touching on the floor and the other leg swinging forward. This posture is similar to a three dimension (3D) inverted pendulum model. This papers presents the dynamic model of a 3D inverted pendulum model and applies to balanced motion planning for a humanoid robot. The obtained results show that the robot is able to keep balance during its movements
\end{abstract}

Keywords. Humanoid robot, inverted pendulum.

\section{INTRODUCTION}

Research on humanoid robots or biped locomotion is currently one of the most exciting topics in the field of robotics and there exist many ongoing projects [1]. One of challenge in motion of a humanoid robot is to keep balance during walking. Many researches concentrate on studying dynamic model of 3D penduluman approximate model in order to apply it into motion of the humanoid robots [2,3]. When humanoid robot walks, almost time of motion is on single phase $[4,5]$. The single phase is characterized by a leg contacting with the ground and the other leg swinging forward. In this case, we can suppose that the reduced model of the plant is similar to a 3D inverted pendulum in kinematics and dynamics. The Three-Dimensional Linear Inverted Pendulum Mode is derived from a general three dimensional inverted pendulum whose motion is constrained to move along an arbitrarily defined plane. It allows a separated controller design for the sagittal $\mathrm{x}-\mathrm{z}$ and the lateral $\mathrm{y}-\mathrm{z}$ motion and simplifies a walking pattern generation a great deal. As analyzied above, we recognize that we can apply the dynamic model of a 3D inverted pendulum into constructing the locomotion gait of a humanoid robot so that the robot can keep balanced posture during walking.

The paper is organized by the following sections: Section 2 presents the the motion equations of a 3D linear inverted pendulum model. Section 3 illustrates the application of the 3D linear inverted pendulum model into planing the walking gait of a humanoid robot. The simulation results are mentioned in section 4 . The conclusion is prensened in section 5 .

\section{MOTION EQUATIONS OF 3D LINEAR INVERTED PENDULUM MODEL}

About structure aspect, 3D Linear Inverted Pendulum Model can be illustrated as Figure 1. Where $r$ is the length of the pendulum shaft, $\beta_{p}$ is the angle between the shaft and $\mathrm{yOz}$ plane, $\beta_{r}$ is the angle between the shaft and $x O y$ plane, $\mathrm{G}(\mathrm{x}, \mathrm{y}, \mathrm{z})$ is the position of the point mass in Cartesian Co-ordinates system, the variables of the pendulum are represented by $q, q=\left(\beta_{r}, \beta_{P}, r\right)$.

where

$$
\begin{gathered}
x=r S_{G}, y=r D, z=-r S_{r} \\
S_{G}=\sin \beta_{P}, \mathrm{~S}_{\mathrm{r}}=\sin \beta_{r}, \mathrm{D}=\sqrt{1-S_{G}^{2}-S_{r}^{2}}, C_{r}=\cos \beta_{r}, C_{G}=\cos \beta_{P}
\end{gathered}
$$

Let $\left(\tau_{r}, f, \tau_{G}\right)$ are actuator torque and force effects on the shaft at $\mathrm{O}$ point, associated with the variables $\left(\beta_{r}, r, \beta_{P}\right) . \mathrm{M}$ is the mass of the pendulum, $\mathrm{g}$ is gravity acceleration. The equation of motion of the 3D inverted pendulum in Cartesian coordinates is given as follows: 


$$
M\left[\begin{array}{c}
\ddot{x} \\
\ddot{y} \\
\ddot{z}
\end{array}\right]=\left(J^{T}\right)^{-1}\left[\begin{array}{c}
\tau_{r} \\
f \\
\tau_{G}
\end{array}\right]+\left[\begin{array}{c}
0 \\
-M g \\
0
\end{array}\right]
$$

So, Jacobian matrix is defined as follows:

$$
J=\frac{\partial p}{\partial q}=\left[\begin{array}{ccc}
0 & S_{G} & r C_{G} \\
-r C_{r} S_{r} / D & D & -r C_{G} S_{G} / D \\
-r C_{r} & -S_{r} & 0
\end{array}\right]
$$

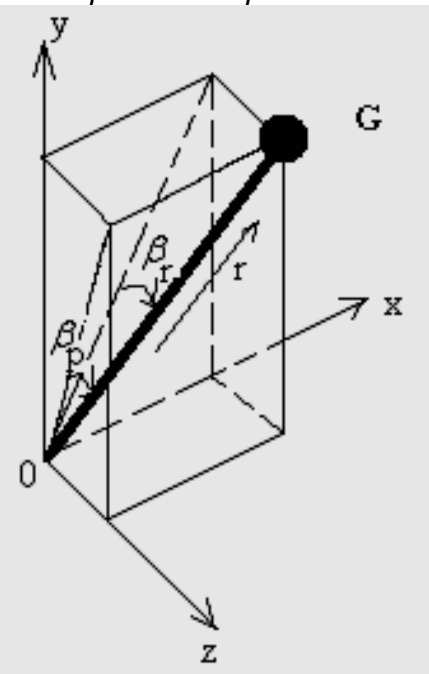

Figure 1: 3D linear inverted pendulum model

Multiply left-hand and right hand side of (3) by $J^{\mathrm{T}}$, we have:

$$
\begin{gathered}
M\left[\begin{array}{ccc}
0 & -r C_{r} S_{r} / D & -r C_{r} \\
S_{G} & D & -S_{r} \\
r C_{G} & -r C_{G} S_{G} / D & 0
\end{array}\right]\left[\begin{array}{c}
\ddot{x} \\
\ddot{y} \\
\ddot{z}
\end{array}\right] \\
\quad=\left[\begin{array}{c}
\tau_{r} \\
f \\
\tau_{G}
\end{array}\right]-M g\left[\begin{array}{c}
-r C_{r} S_{r} / D \\
D \\
-r C_{G} S_{G} / D
\end{array}\right]
\end{gathered}
$$

Using the first row of this equation, we have

$$
M\left(-r D \ddot{z}-r S_{r} \ddot{y}\right)=\frac{D}{C_{r}} \cdot \tau_{r}+r S_{r} M g
$$

By substituting expressions (1) and (2) into (6) we get the equations that describe the dynamics along the $\mathrm{z}$ axis and the $\mathrm{x}$ axis:

$$
\begin{aligned}
& M(-y \ddot{z}-z \ddot{y})=\frac{D}{C_{r}} \cdot \tau_{r}+z M g \\
& M(y \ddot{x}-x \ddot{y})=\frac{D}{C_{G}} \cdot \tau_{g}+x M g
\end{aligned}
$$

Although the moving pattern of the pendulum has vast possibilities but in this research, we want to select a class of motion of the pendulum so that it would be suitable for applying to Robot model. For this reason we apply constraints in order to limit the motion of the pendulum. The first constraint limits the motion in a plane with given normal vector $\left(k_{\mathrm{x},}-1, k z\right)$ and $\mathrm{y}$ intersection at $y_{G}$

$$
y=k_{x} x+k_{z} z+y_{G}
$$

We take the second derivate of (9) as follow:

$$
\ddot{y}=k_{x} \ddot{x}+k_{z} \ddot{z}
$$


We remove y variable from expressions (7), (8), (9), (10), we have:

$$
\begin{aligned}
& \ddot{z}=\frac{z}{y_{G}} g-\frac{k_{x}}{y_{G}}(x \ddot{z}-\ddot{x} z)-\frac{1}{M y_{G}} u_{r} \\
& \ddot{x}=\frac{x}{y_{G}} g+\frac{k_{z}}{y_{G}}(x \ddot{z}-\ddot{x} z)+\frac{1}{M y_{G}} u_{G}
\end{aligned}
$$

where $\tau_{r}=\frac{C_{r}}{D} u_{r}, \tau_{p}=\frac{C_{G}}{D} u_{G}, u_{r}, u_{G}$ are virtual inputs which are introduced to compensate input nonlinearity, In the case of the walking on a plane $\left(k_{x}=k_{z}=0\right)$, and there has no input constraint torque on supporting leg in this research, so $u_{r}=u_{G}=0$. We obtain:

$$
\begin{aligned}
\ddot{z} & =\frac{z}{y_{G}} g \\
\ddot{x} & =\frac{x}{y_{G}} g \\
\text { Set: } \quad \mu & =\sqrt{\frac{g}{y_{G}}}
\end{aligned}
$$

Solving the differential equations (14), (15), we get

$$
\begin{aligned}
& x_{G}=C_{1} e^{-\mu t}+C_{2} e^{\mu t} \\
& z_{G}=C_{3} e^{-\mu t}+C_{4} e^{\mu t}
\end{aligned}
$$

\section{APPLICATION OF THE 3D INVERTED PENDULUM MODEL IN PLANNING THE WALKING GAIT OF A HUMANOID ROBOT.}

\subsection{Center of mass of the robot}

We suppose that we got the center of mass of the robot $G\left(x_{G}, y_{G}, z_{G}\right)$. We consider that the mass of the pendulum and the mass $\mathrm{M}$ are the same, and the shaft which supports $\mathrm{M}$ is one of the two supporting legs of the Robot. So the pendulum which is applied in the Robot model has supporting shaft which changes follows each robot step. This means that the supporting shaft changes continuously from left leg to right leg and vice versa. Base on this, we propose that the robot model includes 7 degrees of freedom (DOF) and the center of mass of each part is located as behind:

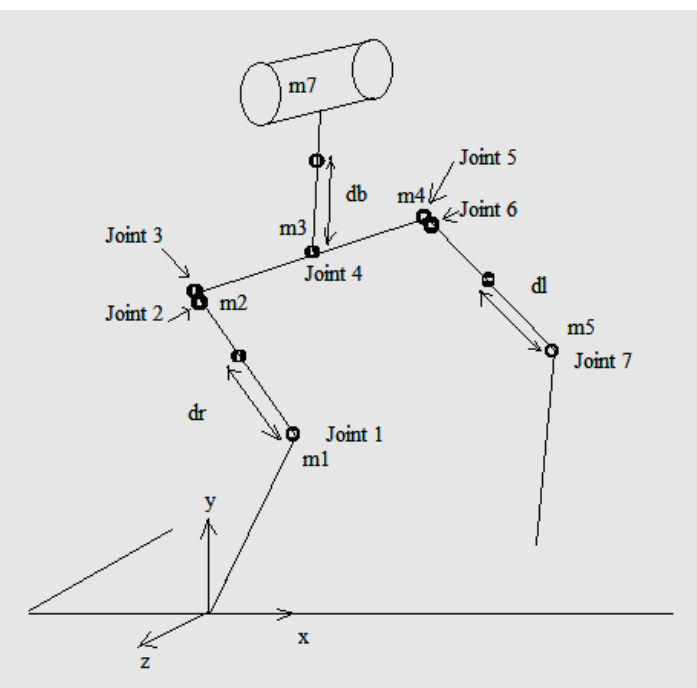

Figure 2: Mass distribution (lateral plane)

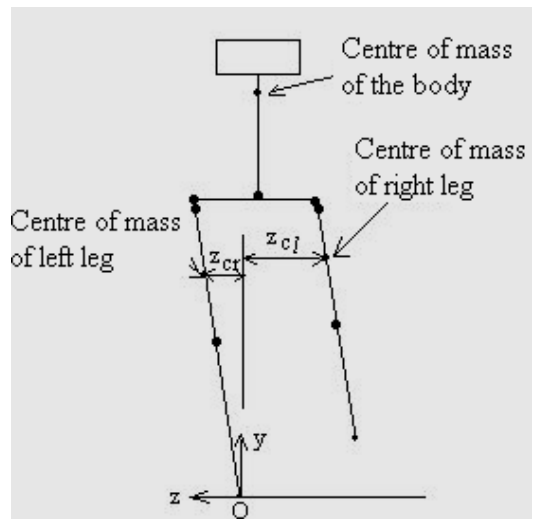

Figure 3: Mass distribution (sagittal plane).

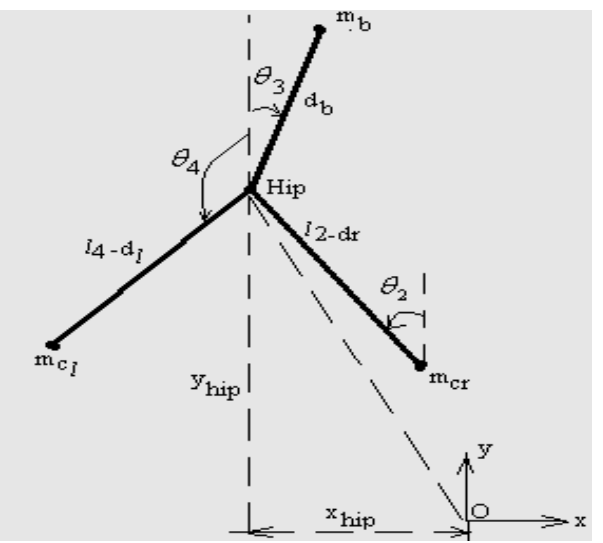

Figure 4: Coordinates of center of mass of each part 
We give a convention of center of mass of each part as follows : Center of mass of part $1 \& 2$ is located at joint 1\&2, part 3's is located at joint 4, part 4's is located at joint 6 , part 5's is located at joint 7. Part 6 is horizontal bar which has a center of mass located at the middle (joint 4), part 7 is homogeneous mass, its center of mass is located at the point which is far from joint 4 by $l_{3}$ distance. By these conventions, we can find the coordinate of the center of mass of each leg and body's of the robot. By synthesizing three coordinates (center of mass of left leg, right leg and body part), we can find out the coordinate of the center of mass of the whole robot system. The figure 4 illustrates this synthesis.

During planning necessary motion pattern of robot model, we need to calculate the center of mass of the whole system and represent it according to the coordinate of robot's hip. We get the coordinate of the center of mass $\mathrm{G}$ after synthesizing the whole robot system.

$$
\begin{gathered}
x_{G}=x_{h}+\frac{m_{b} d_{b}}{2 m_{r}+m_{b}} \sin \theta_{3}+\left(\frac{m_{r}\left(l_{2}-d_{r}\right)}{2 m_{r}+m_{b}}\right) \\
\times\left(\cos \theta_{l} \sin \theta_{4}-\cos \theta_{r} \sin \theta_{2}\right) \\
y_{G}=y_{h}+\frac{m_{b} d_{b}}{2 m_{r}+m_{b}} \cos \theta_{3}+\left(\frac{m_{r}\left(l_{2}-d_{r}\right)}{2 m_{r}+m_{b}}\right) \\
\times\left(\cos \theta_{l} \cos \theta_{4}-\cos \theta_{r} \cos \theta_{2}\right) \\
z_{G}=y_{h} \operatorname{tg} \theta_{r}-(1 / 2) l_{6}+\left(\frac{m_{r}\left(l_{2}-d_{r}\right)}{2 m_{r}+m_{b}}\right) \\
\times\left(\sin \theta_{l} \cos \theta_{4}-\sin \theta_{r} \cos \theta_{2}\right)
\end{gathered}
$$

\subsection{Planning the walking gait of the robot during single phase}

The goal of this section is to plan the walking gait for the robot so that it is similar to human's locomotion. We found that there are two distinct phases in Robot's walking [6]. They are single leg support phase (single phase-SP) and Double leg support phase (double phase-DP). They have a different role. Building each phase in one step so that the robot is not fallen is an important question and also is a big challenge. In the Single Phase, there is only one leg which supports the whole robot, its tip is fixed on the ground, the other swings around the hip to move its tip forward. This action enables the center of mass of the whole robot to move according to the desired walking direction. So, in the Single Phase, the projection of G on the walking plane must has the position which is near the impact point of the supporting leg (Tip_b) on the walking plane to ensure that the robot is always in equilibrium. For the double phase, the system (whole robot) is supported by both legs and enables the robot more stable than the Single Phase does. It means the equilibrium area in Double Phase is bigger than the equilibrium area in the Single Phase on the walking plane. So the double phase is built when the projection of $G$ on the walking plane is far from the impact point of the supporting leg (Tip_b). And the double phase's role is to move the center of mass of robot $(\mathrm{G})$ in during walking. Therefore, the alternation of the single phase and double phase enables the center of mass of the robot $(\mathrm{G})$ both to move forward and to have a trend of moving to positions in the balanced area during walking. This helps robot not to be fallen. To solve the motion planning problem, we built some constraints for the robot, as follows:

1) Direction constraint of the Robot body. We assume that the direction of the body is always vertical is this research. It means that $\theta_{3}$ is always equal to zero. $\theta_{3}=0$

2) Right and left declination constraints of the Robot. When robot walks, right and left declination are equal, $\theta_{r}=\theta_{l}$

3) Constraint of center of mass $G$. From equations (16)(17), establishing the relation between $\mathrm{z}$ and $\mathrm{x}$ direction we get the form of motion curve of the mass $M$ in $x O z$ plane, this curve is similar to the moving pattern of the linear inverted pendulum.

With this motion planning, we have the motion pattern of $\mathrm{G}$ in $\mathrm{xOz}$ as figure 5 . 


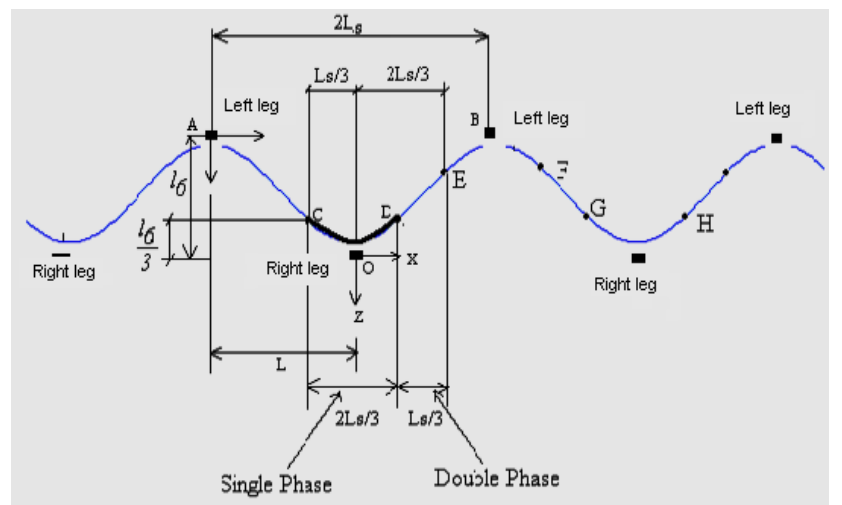

Figure 5: The trajectory of center of mass $\mathrm{G}$

The bold line illustrates the motion pattern of $G$ during sing phase. The slender line illustrates the motion pattern of $G$ during double phase. According to above motion planning, the center of mass of the robot $(\mathrm{G})$ both move forward and has a trend of moving to positions in the stable balanced area during walking. These positions are $\mathrm{O}$ origins which are placed at Tip_b of supporting leg.

From the equations (18), (19), (20) and the constraints 1) and 2), equations of center of mass coordinate calculating are written in more simple form

In order to apply 3D inverted pendulum problem, we must have one more constraint, it is the constraint of the height of $\mathrm{G}$ point. $y_{G}=$ const . This means the center of mass will move in a plane which is parallel with the ground. And the distance between the plane and the ground is $y_{G}, y_{G}$ value was selected in advance.

The selection of parameters as $y_{G}$ and the step distance $L_{s}$ is a big challenge and it is relative selection so that robot is conformable to geometric constraints and has resonable walking gait.

This selection also depends on the robot size, suitable interrelation of parts. The size and mass parameter of the biped robot are given in table below.

Table 1 Dimension and mass of each link

\begin{tabular}{|l|l|l|l|}
\hline$l_{1}$ & $0.175(\mathrm{~m})$ & $\mathrm{m}_{1}$ & $0.5(\mathrm{~kg})$ \\
\hline$l_{2}$ & 0.165 & $\mathrm{~m}_{2}$ & 1 \\
\hline$l_{3}$ & 0.12 & $\mathrm{~m}_{3}$ & 1 \\
\hline$l_{4}$ & 0.165 & $\mathrm{~m}_{4}$ & 1 \\
\hline$l_{5}$ & 0.175 & $\mathrm{~m}_{5}$ & 0.5 \\
\hline$l_{6}$ & 0.1 & $\mathrm{~m}_{6}$ & 0.8 \\
\hline & & $\mathrm{m}_{7}$ & 2.5 \\
\hline
\end{tabular}

According to the table, we get some results, the mass of left leg is equal to the right leg's $m_{r}=m_{l}=1.5$ $\mathrm{kg}$. The mass of the body: $m_{b}=m_{3}+m_{6}+m_{7}=4.3 \mathrm{~kg}$. The mass of the whole robot: $M=m_{r}+m_{l}+m_{b}=7.3$ $\mathrm{kg}$. To solve the problem we still have one more challenge is to select $y_{G}$. If $y_{G}$ is too high, this enables the step distance to be too short, the walking gait will not good. But if $y_{G}$ is too low, this enables the step distance to be too long, the equilibrium of the robot is difficult to achieve. At that time, the actuator torques values at the joints are too big, it is difficult to move center of mass of parts to equilibrium area which is near the impact point of the supporting leg. Figure 6 will illustrates assumed sizes of the robot so that we have a good size selection for the robot.

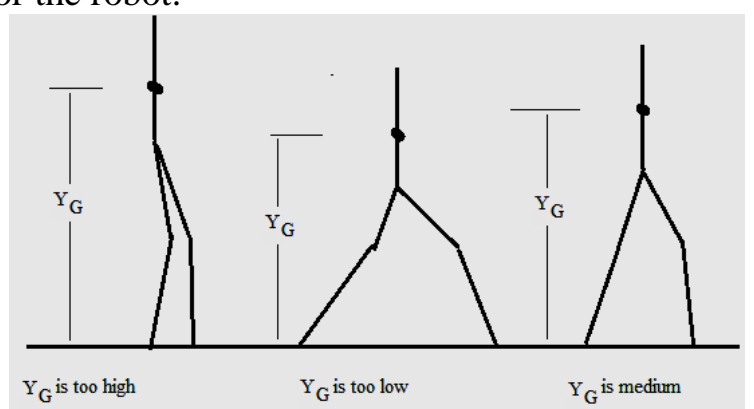

Figure 6: The height of the center of mass $G$ 
We choose relatively $\quad y_{G}=34 \mathrm{~cm}=0.34(\mathrm{~m})$

From (19), we substitute numerical data into it and we get:

$$
\begin{aligned}
& y_{M}=y_{G}=0.34=\left(l_{1} \cos \theta_{1}+l_{2} \cos \theta_{2}\right) \cos \theta_{r} \\
& +0.041+0.0113 \cos \theta_{r}\left(\cos \theta_{4}-\cos \theta_{2}\right)
\end{aligned}
$$

In the single phase, The center of mass $\mathrm{G}$ moves on CD curve which is near the Tip_b point of supporting leg (O origin). We choose one more parameter $L_{s}=0.09(\mathrm{~m})$ (is the chosen step distance) with a scale which is suitable for the height of the robot. The time during the Single Phase is chosen $T_{s}=2 \mathrm{~s}$. The time origin was chose at the time which the center of mass $G$ is stay at $C$ point, Completing the single phase at the time $t=T_{s}$ when $\mathrm{G}$ is stay at D. DE curve illustrates the double phase. EF curve illustrates the single phase in the next step. At this moment the supporting leg is left leg and we have to set the time origin again $t=0$ when $\mathrm{G}$ is stay at $\mathrm{E}$. Completing the single phase in this step cycle at the time $t=T_{s}\left(0 \leq t \leq T_{s}\right)$ when $\mathrm{G}$ is stay at $\mathrm{F}$. Note that the time origin is always set again after completing each single phase and the coordinate origin $\mathrm{O}$ is always set when the robot changes supporting leg. From initial constraints of $\mathrm{G}$ in the single phase together with (16) (17), we found out coefficients.

$C_{l}=-0.03 ; C_{2}=0.00000065 ; C_{3}=-0.03299 ; C_{4}=-0.000000712 ; \mu=\sqrt{\frac{g}{y_{G}}}=\sqrt{\frac{9.81}{0.34}}=5.37$.

In summary, there are 3 constraints of moving of $\mathrm{G}$ :

4) Constraint of end point of the swing leg (Tip_e)

$$
\left\{\begin{array}{l}
x_{G}=-0.03 \cdot e^{-5.37 . t}+0.00000065 . e^{5.37 . t} \\
=\left(l_{1} \sin \theta_{1}+l_{2} \sin \theta_{2}\right) \cos \theta_{r} \\
+0.0113 \cos \theta_{r}\left(\sin \theta_{4}-\sin \theta_{2}\right) \\
y_{G}=0.34=\left(l_{1} \cos \theta_{1}+l_{2} \cos \theta_{2}\right) \cos \theta_{r} \\
+0.041+0.0113 \cos \theta_{r}\left(\cos \theta_{4}-\cos \theta_{2}\right) \\
z_{G}=-0.03299 \cdot e^{-5.37 . t}-0.00000072 . e^{5.37 . t} \\
=\left(l_{1} \cos \theta_{1}+l_{2} \cos \theta_{2}\right) \cos \theta_{r} \cdot \operatorname{tg} \theta_{r} \\
-0.05+0.0113 \sin \theta_{r}\left(\cos \theta_{4}-\cos \theta_{2}\right)
\end{array}\right.
$$

Surveying a humanoid walking gait, we try design so that $x_{e}=f_{x}(t)$ is a third polynomial function respect with $\mathrm{t}$ and $y_{e}=f_{y}(t)$ is a fifth polynomial function respect with $\mathrm{t}$. Coefficients of polynomial functions are define from initial constraints as the figure below, and using this constraint to find out two constraints of Tip_e:

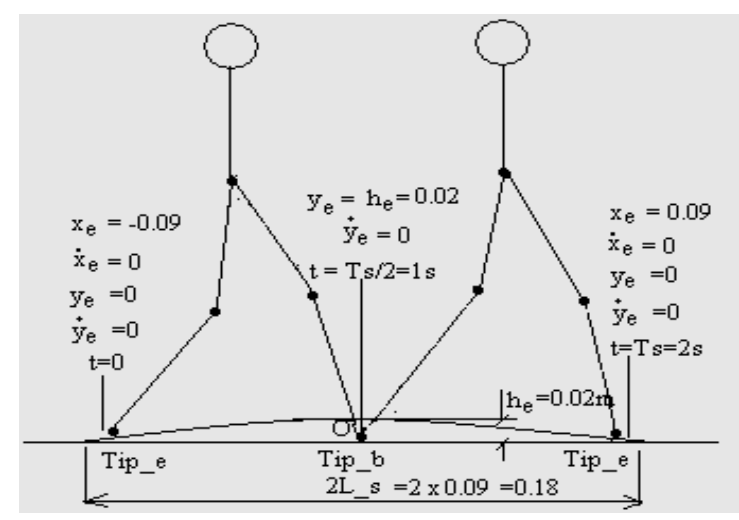

Figure 7: Definition of the initial constraint of Tip e 


$$
\begin{aligned}
& x_{e}=\left(l_{1} \sin \theta_{1}+l_{2} \sin \theta_{2}+l_{4} \sin \theta_{4}+l_{5} \sin \theta_{5}\right) \\
& \times \cos \theta_{r}=-0.09+0.135 t^{2}-0.045 t^{3} \\
& y_{e}=\left(l_{1} \cos \theta_{1}+l_{2} \cos \theta_{2}+l_{4} \cos \theta_{4}+l_{5} \cos \theta_{5}\right) \\
& \times \cos \theta_{r}=0.08 t^{2}-0.08 t^{3}+0.02 t^{4}
\end{aligned}
$$

From the three constraints of $\mathrm{G}$ and two constraints of Tip_e, we found out five constraints to find out angles $\theta_{1}, \theta_{2}, \theta_{4}, \theta_{5}, \theta_{r}$, and $\theta_{3}=0, \theta_{r}=\theta_{l}$.

\subsection{Planning the walking gait of the robot during double phase}

In double phase, both legs of the robot impact with the ground to support the body and carry the center of mass forward. This helps the robot not to be fallen when its centcenter of mass is far from the supporting leg. 1) Direction constraint of the Robot body. For the biped robot, we assume that the direction of the body is always vertical in this research. It means that $\theta_{3}$ is always equal to zero. $\theta_{3}=0$

2) Right and left declination constraints of the robot. Similarly in single phase, when the robot walks, right and left declination are equal, $\theta_{r}=\theta_{l}$

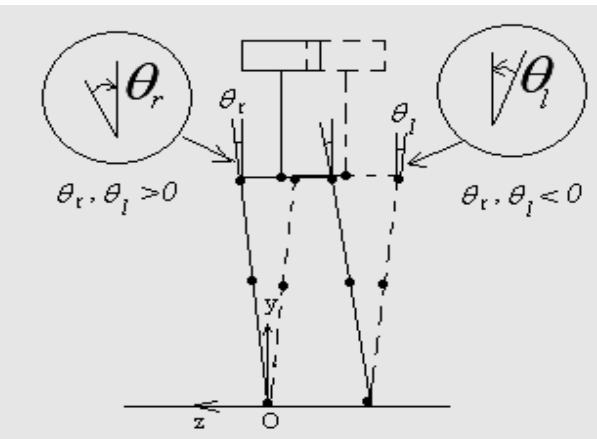

Figure 8: Robot leans to the right and left side

In Fiure 8, the robot is leaning to the right (the slender continuous line), The center of mass is concentrating on the right leg. In the double phase, the robot both moves forward and leans to the left to concentrate the center of mass on the left leg and to carry out the single phase in the next step. The equation $\theta_{r}=\theta_{l}$ ensures that the robot has equilateral lean on both legs.

3) Constraint of center of mass $G$ for the humanoid robot

To ensure the continuity after completing the single phase and beginning the double phase, it is necessary to have a continuity of geometry at positions $\mathrm{D}$ and $\mathrm{E}$ so that the trajectory of the center of mass $\mathrm{G}$ is continuous. Therefore, $y_{G}=0.34(\mathrm{~m})$

We design the trajectory from $\mathrm{D} \rightarrow \mathrm{E}$ so that the center of mass moves on a straight line $\mathrm{DE}$ with $x_{G}=$ $a_{0}+a_{l} t$ and $z_{G}=b_{0}+b_{1} t\left(0 \leq t \leq T_{D}\right)$.

We have 3 constraints of the center of mass $G$ and some initial conditions are shown in Figure 9:

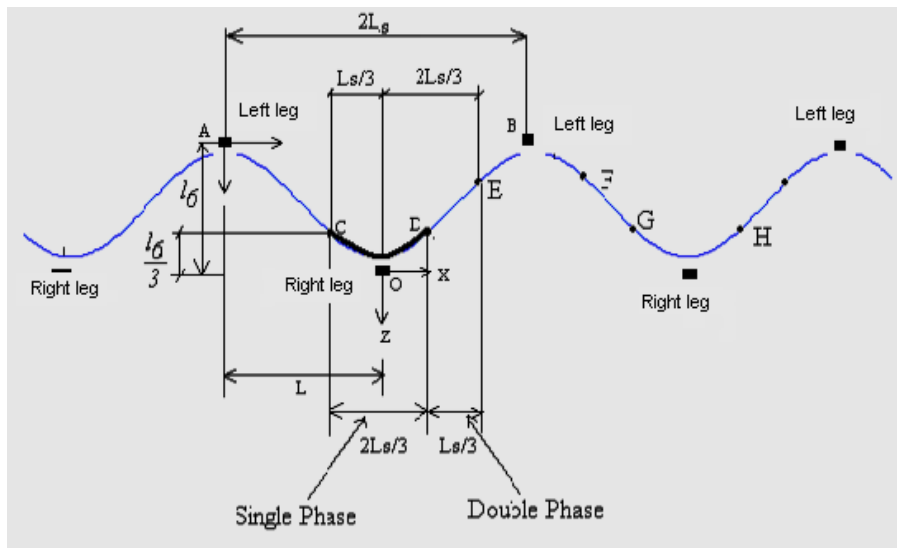

Figure 9: Initial conditions of Double Phase 
4) Constraint of Tip_e

$$
\left\{\begin{array}{l}
x_{G}=0.03+0.16115 t=\left(l_{1} \sin \theta_{1}+l_{2} \sin \theta_{2}\right) \cos \theta_{r} \\
+0.0113 \cos \theta_{r}\left(\sin \theta_{4}-\sin \theta_{2}\right) \\
y_{G}=y_{G}=0.34=\left(l_{1} \cos \theta_{1}+l_{2} \cos \theta_{2}\right) \cos \theta_{r} \\
+0.041+0.0113 \cos \theta_{r}\left(\cos \theta_{4}-\cos \theta_{2}\right) \\
z_{G}=-0.0333-0.17651 t=\left(l_{1} \cos \theta_{1}+l_{2} \cos \theta_{2}\right) \\
\times \cos \theta_{r} \cdot \operatorname{tg} \theta_{r}-0.05+0.0113 \sin \theta_{r}\left(\cos \theta_{4}-\cos \theta_{2}\right)
\end{array}\right.
$$

Because the impact points of both legs are two fixed points on the ground so we have: $x_{e}=L_{s}=0.09(m)$ ( $L_{\mathrm{s}}$ is the distance of each step) and $y_{e}=0$. We find out the constraint for the Tip_e as follows:

$$
\left\{\begin{array}{l}
x_{e}=\left(l_{1} \sin \theta_{1}+l_{2} \sin \theta_{2}+l_{4} \sin \theta_{4}+l_{5} \sin \theta_{5}\right) \\
\times \cos \theta_{r}=0.09 \\
y_{e}=\left(l_{1} \cos \theta_{1}+l_{2} \cos \theta_{2}+l_{4} \cos \theta_{4}+l_{5} \cos \theta_{5}\right) \\
\times \cos \theta_{r}=0
\end{array}\right.
$$

Similarly in Single Phase, from the three constraints of $\mathrm{G}$ and two constraints of Tip_e, we found out five constraints to find out angles $\theta_{1} \cdot \theta_{2} \cdot \theta_{3} \cdot \theta_{4} \cdot \theta_{5} \cdot \theta_{r}$ and $\theta_{1}=0, \theta_{r}=\theta_{l}$.

\section{SIMULATION RESULTS}

Using Matlab software, building block diagram by Simulink for the constraints above we find angles $\theta_{1}, \theta_{2}, \theta_{4}, \theta_{5}, \theta_{r}$ respect with time t. Some obtained simulation results when solve the motion planning by Matlab software is:

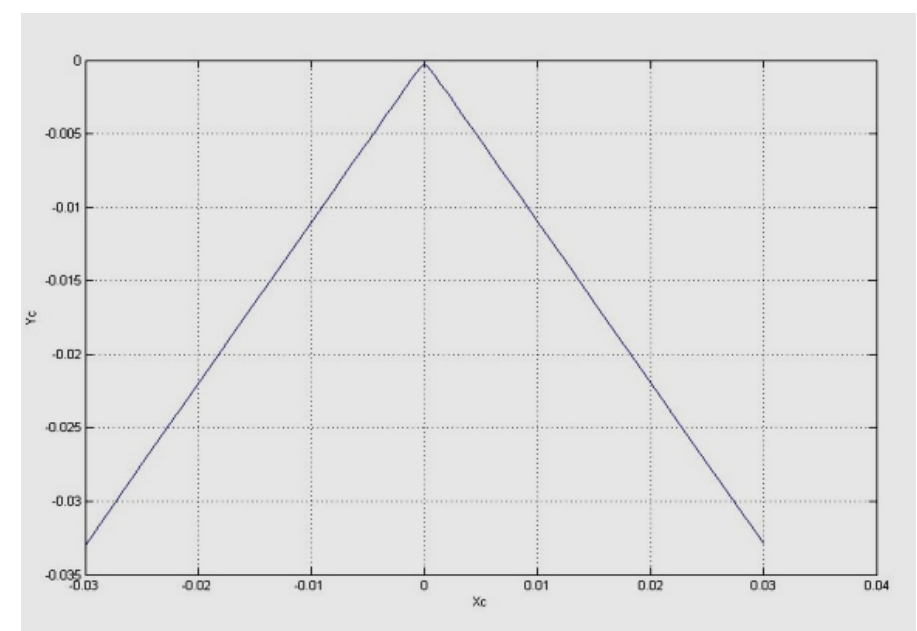

Figure 10: $\mathrm{XG}, \mathrm{ZG}$ in the single phase

We see that the result in figure 10 is similar to the black bold curve in the swing phase. That curve shows the movement of the center of mass $\mathrm{G}$ in the swing phase. This movement is kept in the stable equilibrium area of the robot. 


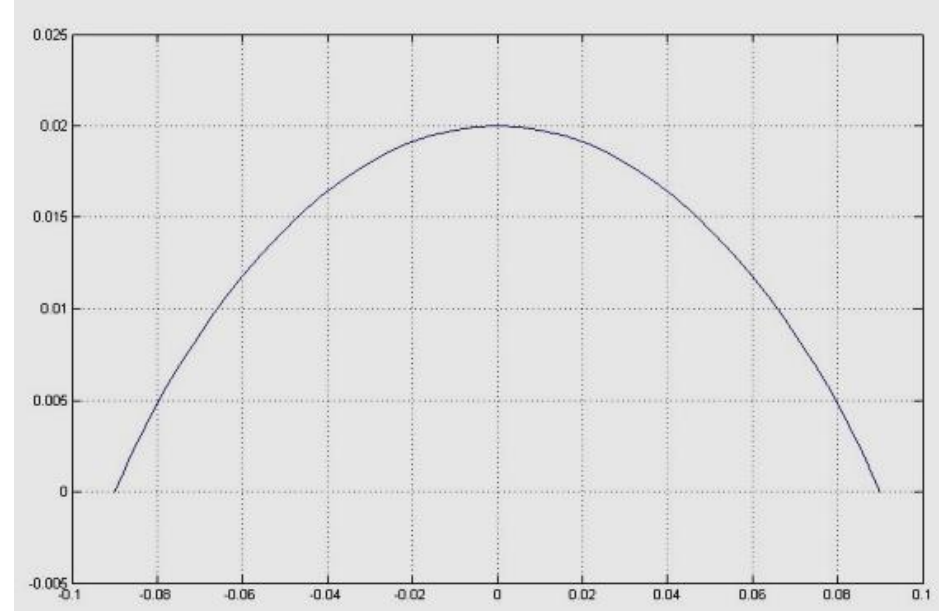

Figure 11: XE, YE in the single phase

The result in Figure 11 shows that the Tip E of the swing leg is moved forward by lifting the tip far from the ground.

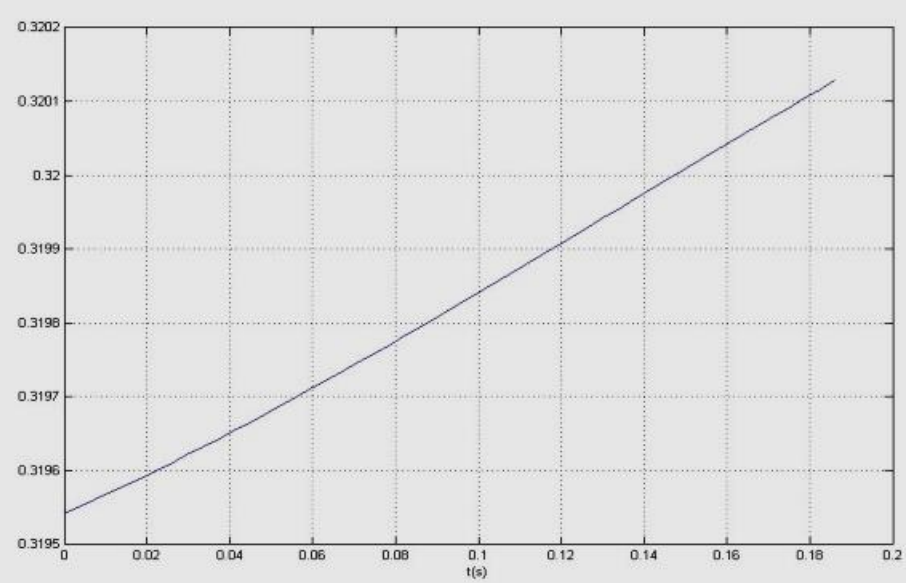

Figure 12: Xhip Yhip in the double phase

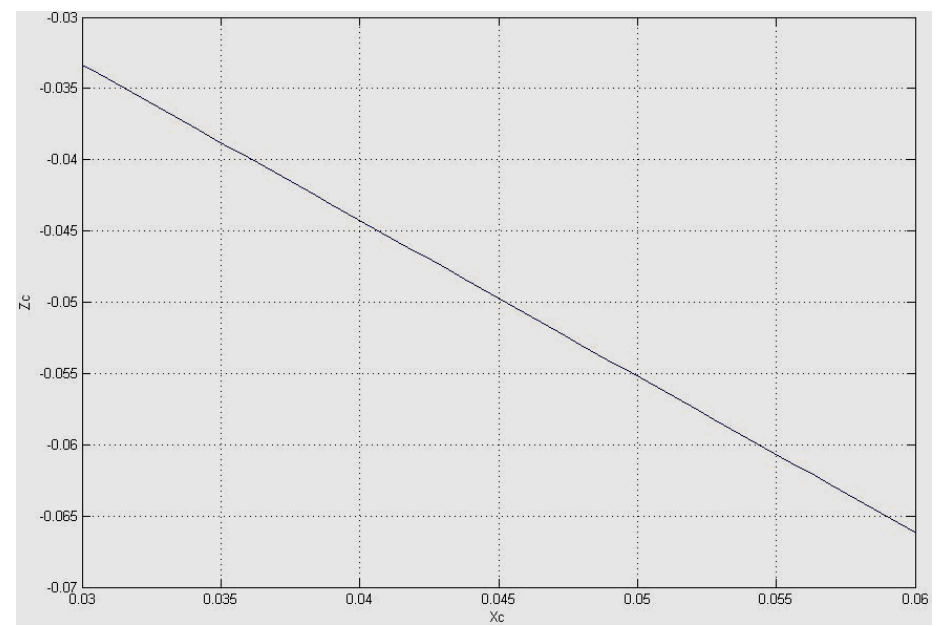

Figure 13: $\mathrm{X}_{\mathrm{G}}, \mathrm{Z}_{\mathrm{G}}$ in the double phase

The results in Figure 12 and 13 illustrate the movements of thie hip point and the center of mass $G$ in the double phase. These movement is be sured in the equilibrium area. 


\section{CONCLUSION}

The paper is researched the 3D inverted pendulum model in order to apply into motion planning for a humanoid robot. The achieved results show that the center of mass of the robot is alway in the stable area. This prevents the robot from falling down. In additon, this motion planning also helps the robot move in the manner that is similar to the human locomotion. Based on the results together with kinematics model and dynamics model, we will design a robust damping control for the robot. We plan to build a real mechanical model in future.

\section{REFERENCES}

[1] Y. Liu and et al., Human-Like Walking with Heel Off and Toe Support for Biped Robot, Applied Sciences, Vol 7, pp.499, 2017

[2] M. S.Ashtiani and et al., Robust Bipedal Locomotion Control Based on Model Predictive Control and Divergent Component of Motion, IEEE ICRA, 2017

[3] S.Kajita et al, 3D Linear Inverted Pendulum Mode: A simple modeling for a Biped walking parttern generation, International Conference on Intelligent Robots and Systems, 2001.

[4] F. Zonfrilli, Theoretical and Experimental Issues in Biped Walking Control Based on Passive Dynamics, PhD thesis, 2004.

[5] J. Denk and G. Schmidt, Synthesis of a Walking Primitive Database for a Humanoid Robot using Optimal Control Techniques, Proceedings of IEEE-RAS International Conference on Humanoid Robots (HUMANOIDS2001) Tokyo, Japan, pp. 319-326, November 2001

[6] A.Kharb and et al, A review of gait cycle and its parameters, IJCEM, Vol 13, 2011

\section{HOẠCH ĐỊNH CHUYỂN ĐộNG CÂN BẦNG CHO ROBOT HÌNH NGƯờI DỰA VÀO MÔ HÌNH CON LẮC NGƯợC 3 CHIỀU}

Tóm tắt. Các nghiên cứu về robot hình ngoài luôn luôn thu hút nhiều nhà nghiên cứu trong lĩnh vực robot. Một trong những thách thức của robot hình người đó là giữ robot di chuyển cân bằng và ổn định. Vì các robot hình người luôn di chuyển bằng hai chân, hầu hết thời gian trong chu kỳ bước của robot hình người là một chân chạm đất và chân còn lại di chuyển trên không về phía trước. Dáng điệu này tương tự như mô hình của con lắc ngược 3 chiều. Bài báo này giới thiệu mô hình động lực học của con lắc ngược 3 chiều và áp dụng mô hình nào vào việc hoạch định di chuyển cân bằng cho robot hình người. Các kết quả thu được cho thây rằng robot có khả năng giữ cân bằng trong quá trình chúng chuyển động.

Từ khóa: Robot hình người, mô hình con lắc ngược

Ngày nhận bài: $30 / 10 / 2018$

Ngày chấp nhận đăng: 20/05/2019 